\title{
Tilted Fluctuation Electron Microscopy Characterization of Magnetically Anisotropic Amorphous Metal Films
}

\author{
Ellis Kennedy ${ }^{1 *}$, Alejandro Ceballos ${ }^{1,3}$, Frances Hellman $^{1,2,3}$, Colin Ophus $^{4}$ and Mary Scott ${ }^{1,4}$ \\ 1. Department of Materials Science and Engineering, University of California, Berkeley, Berkeley, CA, \\ USA. \\ 2. Department of Physics, University of California, Berkeley, Berkeley, CA, USA. \\ 3. Materials Sciences Division, Lawrence Berkeley National Lab, Berkeley, CA, USA. \\ 4. The Molecular Foundry, Lawrence Berkeley National Lab, Berkeley, CA, USA. \\ * Corresponding author: ellisrae@berkeley.edu
}

Transmission electron microscope (TEM) characterization of sputtered amorphous $\mathrm{Tb}_{\mathrm{x}} \mathrm{Co}_{1-\mathrm{x}}(a-\mathrm{Tb}-\mathrm{Co})$ thin films is used to better understand the magnetic anisotropy exhibited by the metal films under certain growth conditions, compositions, and post-deposition heat treatment. This work aims to link observed changes in the magnetic properties of amorphous $\mathrm{Tb}_{\mathrm{x}} \mathrm{Co}_{1-\mathrm{x}}(a-\mathrm{Tb}-\mathrm{Co})$ to medium range (on the order of 2$4 \mathrm{~nm}$ ) structural changes. It is thought that the magnetic anisotropy originates from the single ion anisotropy of $\mathrm{Tb}$ together with an anisotropic local pair-ordering induced by the growth process, but how the magnetic properties of this material depend on structure, and why it depends on both growth and annealing temperatures, remain unexplained $[1,2]$. Understanding the effects of growth conditions on atomic ordering will allow fine tuning of the material structure in order to produce the desired magnetic properties. These films are one of many transition metal and rare earth element alloys that have desirable magnetic properties for application in memory storage devices.

Amorphous $\mathrm{Tb}_{\mathrm{x}} \mathrm{Co}_{1-\mathrm{x}}(a-\mathrm{Tb}-\mathrm{Co})$ oxidizes rapidly with exposure to air and must be capped with a protective layer. Films with aluminum oxide, tantalum oxide, and $\mathrm{SiN}_{\mathrm{x}}$ protective layers were studied using scanning nanodiffraction in the TEM. Aluminum oxide was determined to be polycrystalline when viewed in diffraction and tantalum oxide produced an amorphous ring pattern that obscured that amorphous $\mathrm{Tb}_{\mathrm{x}} \mathrm{Co}_{1}$ $\mathrm{x}(a-\mathrm{Tb}-\mathrm{Co})$ signal making analysis difficult. $\mathrm{SiN}_{\mathrm{x}}$ was selected because of its robustness as a capping layer and because the $\mathrm{SiN}_{\mathrm{x}}$ signal does not interfere with the signal from the first ring detected from $\mathrm{Tb}_{\mathrm{x}} \mathrm{Co}_{1-\mathrm{x}}(a-\mathrm{Tb}-\mathrm{Co})$ using scanning nanodiffraction. The primary method of characterizing the metallic films is fluctuation electron microscopy (FEM). FEM is a focused-probe, scanning nanodiffraction technique that is sensitive to medium range order in disordered materials. By measuring a series of diffraction patterns taken over a small region of the film and calculating the variance in intensity as a function of spatial frequency, FEM has been used to determine the relative amount of medium range ordering on the size scale of the probe used [3]. Typically, FEM experiments assume a sample is structurally anisotropic. We have extended FEM to probe directionally-dependent properties of anisotropic materials by tilting a single sample between sequential data scans. Since diffracted intensity depends most strongly on periodic structures that lie in the plane perpendicular to the beam direction, this method probes in-plane and out-of-plane structures as the sample's tilt is changed. This allows for comparison to in-plane and out-of-plane variance to determine differences in medium range order in these directions. $\mathrm{Tb}_{\mathrm{x}} \mathrm{Co}_{1-\mathrm{x}}(a-\mathrm{Tb}-\mathrm{Co})$ thin films have a magnetic easy axis perpendicular to the plane of the film, so tilting with respect to the easy axis provides a method of relating magnetic and structural properties [4]. 
The $\mathrm{SiN}_{\mathrm{x}}$ capping allows for more thorough analysis of variance peak features and easier full width at half maximum (FWHM) comparisons. To calculate precise variance values, a fitting protocol was developed to remove ellipticity resulting from imperfect microscope alignments. Figure 1 shows the custom data processing steps and elliptical fitting that was performed before the variance was calculated. The fitted and corrected diffraction patterns were analyzed to determine differences in variance as a function of spatial frequency with respect to scattering angle. Figure 2 shows representative tilted FEM data collected from a $\mathrm{Tb}_{\mathrm{x}} \mathrm{Co}_{1-\mathrm{x}}(a-\mathrm{Tb}-\mathrm{Co})$ film grown at room temperature and capped with $\mathrm{SiN}_{\mathrm{x}}$. The FWHM statistics and features of the angle-resolved variance peaks will be used for characterization of structural dependence on direction within the metal film. By relating changes in MRO to changes in the magnetic properties arising from thermal annealing and growth conditions, one can better understand the atomicscale structural origins of magnetic anisotropy in this system. The tilted FEM procedure offers a novel method of characterizing directionally-dependent ordering in thin films and elucidates how changes in an amorphous structure are linked to material properties [5].

\section{References:}

[1] S Yoshino et al., Japanese Journal of Applied Physics 23(2R) (1984), p.188.

[2] F Hellman and EM Gyorgy, Physical Review Letters 68.9 (1992), p. 1391.

[3] PM Voyles and DA Muller, Ultramicroscopy 93(2) (2002), p.147.

[4] T Ueno et al., Journal of Physics D: Applied Physics 49(20) (2016), p.205001.

[5] This project is supported by the U.S. Department of Energy, Office of Science, Office of Basic

Energy Sciences, Materials Sciences and Engineering Division under Contract No. DE-AC02-05-

CH11231 within the Non-equilibrium Magnetic Materials Program. Work at the Molecular Foundry was supported by the Office of Science, Office of Basic Energy Sciences, of the U.S. Department of Energy under Contract No. DE-AC02-05CH11231.

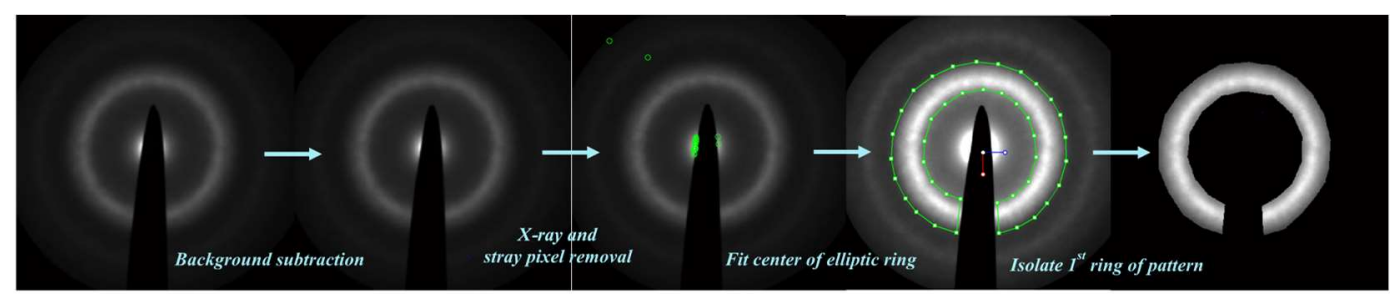

Figure 1. FEM processing steps and elliptical fitting of 4D scanning nanodiffraction data that identifies the diffracted ring and corrects ellipticity resulting from inevitable probe stigmation.

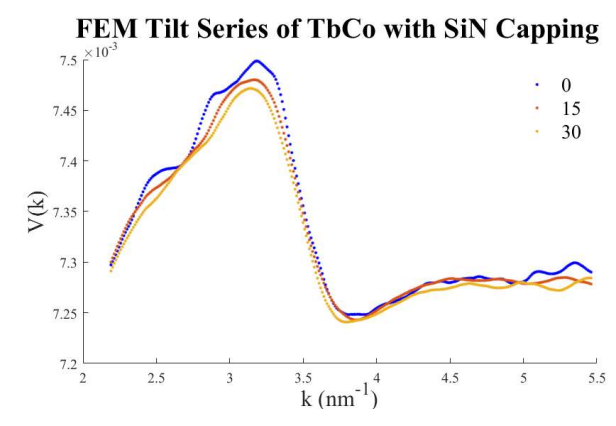

Figure 2. Tilted FEM data collected from a $\mathrm{Tb}_{\mathrm{x}} \mathrm{Co}_{1-\mathrm{x}}(a-\mathrm{Tb}-\mathrm{Co})$ film tilted to $0^{\circ}, 15^{\circ}$, and $30^{\circ}$. 